\title{
Disarming smiles: irrelevant happy faces slow post-error responses
}

\author{
Rashmi Gupta $^{1,2} \cdot$ Gedeon O. Deák $^{2}$
}

Received: 1 September 2014 / Accepted: 27 June 2015/Published online: 21 July 2015

(C) Marta Olivetti Belardinelli and Springer-Verlag Berlin Heidelberg 2015

\begin{abstract}
When we make errors, we tend to experience a negative emotional state. In addition, if our errors are witnessed by other people, we might expect those observers to respond negatively. However, little is known about how implicit social feedback like facial expressions influences error processing. We explored this using the cognitive control phenomenon of post-error slowing: the tendency to slow the response immediately following an error. Adult participants performed a difficult perceptual task: estimating which of two lines (horizontal or vertical) was longer. The background showed an irrelevant distractor face with a happy, sad, or neutral expression. Participants slowed after errors only when the subsequent distractor face was happy, but not when the subsequent distractor was sad or neutral nor when a happy face followed a correct response. This suggests that information about others' affect, even non-interactive, task-irrelevant information, has performance- and valence-dependent effects on adaptive cognitive control.
\end{abstract}

Keywords Cognitive conflict - Emotional expression . Error processing - Face processing · Post-error slowing . Social feedback

Rashmi Gupta

rash_cogsci@yahoo.com

1 Swiss Center for Affective Sciences, University of Geneva, Campus Biotech, 9, Chemin des Mines, 1202 Geneva, Switzerland

2 Department of Cognitive Science, University of California, San Diego, CA, USA

\section{Introduction}

Error monitoring refers to the online detection of errors in order to generate internal feedback signals that can contribute to adaptive performance modification. It is therefore an executive control process that facilitates top-down adjustment of cognitive processes and behavioural decisions (Norman and Shallice 1986). A behaviour believed to reflect error monitoring is the slowing of responses following errors, or post-error slowing (i.e. PES). PES has been observed in speeded reaction time tasks, reasoning tasks, verbal analogy tasks, memory search tasks, and response-inhibition tasks (see Botvinick et al. 2001; Danielmeie and Ullsperger 2011). It has even been reported in preschool-aged children (Jones et al. 2003), suggesting that PES is a robust, general control mechanism for performance adaptation.

Several accounts have been proposed to explain PES (Danielmeie and Ullsperger 2011). One idea is that PES is related to cognitive control processes that are initiated following the commission of an error, and therefore, it is specifically related to error processing (Botvinick et al. 2001). A distinct alternative hypothesis is that PES reflects an orienting response following infrequent events such as errors, and therefore is not necessarily tied to error processing (Notebaert et al. 2009). A third hypothesis is that PES reflects motor inhibition processes that serve cognitive control (Ridderinkhof 2002; Marco-Pallares et al. 2008). These hypotheses are not mutually exclusive, but all of them focus on cognitive control processes. However, it is possible that additional emotional and social factors influence PES. If this is true, it could demonstrate that emotional or evaluative processes are components or moderators of PES.

In everyday situations, when we make errors, various environmental cues might help us recognize and evaluate 
our mistakes, so that we can adapt our responses in future similar situations. Everyday situations entail rich social contexts that may include real or imagined others who can observe and evaluate our actions. Thus, not only do we evaluate our own performance, but we also expect others to evaluate our performance, at least sometimes. These evaluations can be made explicit through social feedback: behavioural cues that reflect the evaluator's attitude, and that may indirectly influence how we assess and adjust our performance.

Social feedback can take a variety of forms: teachers mark students' papers, parents reward or punish their children's behaviours, employers give employees performance evaluations, etc. Some forms of feedback, however, are less explicit. For example, facial expressions are a salient kind of social information that may serve as performance feedback (Herba and Phillips 2004; for a review see Gao and Maurer 2009). Infants develop the ability to discriminate facial expressions within the first few weeks (Farroni et al. 2007), and by 6 months, they begin adapting behaviourally to parents' facial expression (Walden and Ogan 1988). Thus, within our first year, we use facial expressions as differential behavioural signals. By adulthood, we can process facial expressions extremely quickly and use them to modify attitudes and responses (Batty and Taylor 2003; Pizzagalli et al. 2002; Winkielman et al. 1997).

There is a close relationship between error processing and affect (Korban and Pourtois 2014; Shackman et al. 2011). According to appraisal theories, our emotions are elicited by events that are relevant to us, including our own actions (Scherer 2001; Schacht et al. 2010; Picton et al. 2012). Our errors can elicit error-related neural processing (Gehring et al. 1993) and negative emotional reactions. Moreover, in some situations, we expect our errors to elicit others' negative evaluations and responses (e.g. Funder 1987; Schlenker and Leary 1982). This social error feedback may be communicated through explicit messages or through implicit cues such as facial expressions. However, little is known about how we process implicit social feedback. One complication is the ubiquity of facial expressions: we frequently see others' facial expressions, but they are often unrelated to the accuracy or quality of our actions. Thus, although facial expressions can serve as feedback, they typically do not. Nevertheless, because facial affect processing is to some extent automatic (Jiang and He 2006; Vuilleumier et al. 2001), others' facial expressions might be implicitly interpreted as feedback, even when unrelated to our performance. If this is true, then others' displays of facial affect will sometimes be incongruent with our own error-monitoring evaluation. Such incongruent feedback might elicit cognitive conflict, and subsequent processing to resolve the conflicting signals from our internal error monitoring, and implicit feedback from the other's emotional cues. For example, if we happen to see someone smiling after we make an error, or see a sad face after performing well, the apparently conflicting feedback signals might require extra time and cognitive resources to resolve. In this light, smiles might not always serve as positive or helpful feedback.

In the present study, we examined how PES may be influenced by implicit emotional feedback. Adult participants performed a challenging speeded perceptual task in which they judged which of two lines (horizontal or vertical) was longer. The task, though simple, was subtle enough to elicit errors. A static face, which was irrelevant to the task (i.e. a distractor), was displayed in the background in every trial. This task-irrelevant distractor face exhibited a happy, sad, or neutral expression. Because the facial expression on any trial was random-not dependent on the participant's performance-each correct response or error was followed, on the next trial, by an incidental happy, sad, or neutral face which, in some trials, created an implicit mismatch that could be interpreted by automatic processes as a conflicting error signal. For example, a sad face could follow a correct response, or a happy face could follow an error, and in such trials, the face constituted implicit incongruent feedback. By contrast, a happy face following a correct response or sad face following an error constituted implicit congruent feedback. Importantly, facial expressions were implicit, not explicit, feedback, because participants knew that the faces were unrelated to their accuracy.

We investigated whether participants would show more PES when a happy face appeared after an error, than when other facial expressions followed errors. If so, it might indicate that happy faces act as automatically processed feedback cues that are compared to internal error-monitoring signals. An alternative possibility is that participants also will show similar PES when a sad face follows a correct response. This would suggest that any discrepancy between internal error signals and social cues can increase processing costs and cause slowing. However, because PES typically occurs after error trials, we might further predict relatively more slowing after error trials with discrepant happy faces, than after correct trials followed by discrepant sad faces. A third possibility is that PES is independent of implicit feedback: that is, if conflict processing is entirely or overwhelmingly due to endogenous error signals, not social feedback, then PES should occur after error trials, regardless of the expression of the following face. Conversely, the fourth possibility is that the emotional expression of the post-response face might affect response speed on that trial, independent of previous-trial accuracy. That is, perhaps happy faces generally accelerate responses and sad faces generally slow responses (akin to 
encouragement or discouragement). This effect could also be independent of PES: that is, PES might occur after error trials, and there might be an overarching main effect of expression, with slower performance on sad-face than on happy-face trials.

\section{Methods}

\section{Participants}

In exchange for course credit, 20 North American, Englishfluent (Caucasian) right-handed college students (10 males, mean age $=21.8$ years) with normal or corrected-to-normal vision participated. Participants were all enrolled in psychology or cognitive science classes. Procedures for obtaining informed consent, and all experimental procedures, adhered to university IRB policies.

\section{Apparatus}

Stimuli were displayed on a 13-in. colour monitor viewed from a distance of $60 \mathrm{~cm}$. E-Prime software (Version 1.1) (Schneider et al. 2002) was used to present stimuli.

\section{Stimuli}

Eight upright greyscale faces $\left(11.6^{\circ} \times 10.9^{\circ}\right)$ of young adults (four males, four females) bearing either a happy, sad, or a neutral expression, served as distractors. Faces were selected from the Karolinska Directed Emotional Face database (Lundqvist et al. 1998). A red cross was superimposed on the forehead of each face, with one line slightly longer than the other. Length differences were chosen (based on pretesting) to be difficult to discriminate when lines were at different angles. The lengths of linepairs were (in visual angle) $1.9^{\circ}$ versus $2.38^{\circ}, 1.52^{\circ}$ versus $1.90^{\circ}$, and $1.14^{\circ}$ versus $1.43^{\circ}$. Each of the line-pairs had a 5:4 ratio between the longer and shorter line. An example is shown in Fig. 1. Lines were positioned and scaled, relative to the face, so that top-down or configural cues such as the distance of lines from facial landmarks would be virtually impossible to use. In addition, participants could not use facial information to perform the task because the orientation and size of the longer line was randomized across trials, so distances to facial features were constantly changing. Further, different faces were used in each block, so participants could not learn to use specific facial features as landmarks. Finally, the presentation was so rapid that top-down spatial strategies were not feasible. These aspects of the design are important, because if participants could have used facial cues to facilitate task performance,

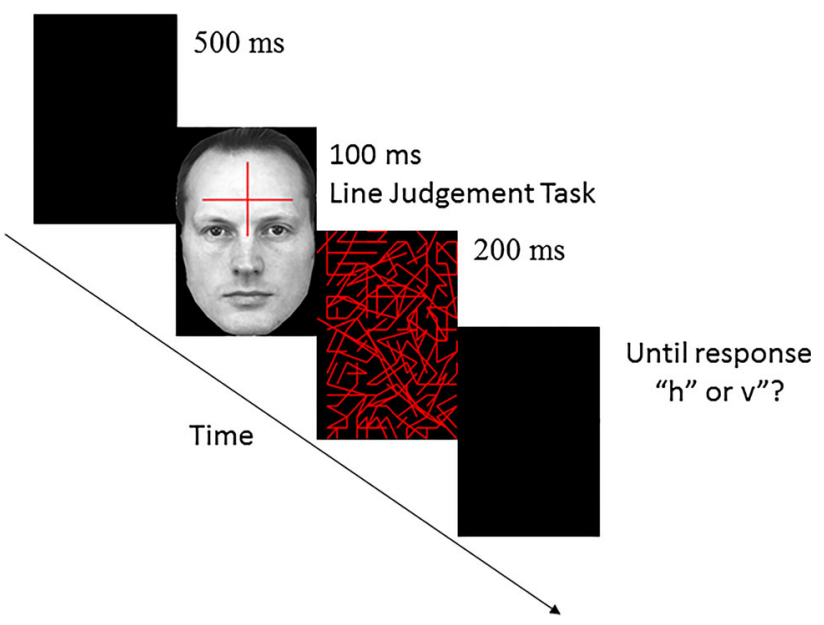

Fig. 1 Schematic sequence of events in an example trial

we could not assume that faces provided strictly implicit information.

\section{Experimental design and procedure}

Each trial began with the presentation of a $500 \mathrm{~ms}$ central fixation point, followed by a $100 \mathrm{~ms}$ stimulus display, and then a $200 \mathrm{~ms}$ visual mask (i.e. red pattern on black background; see Fig. 1). The brevity of the display both precluded top-down uses of facial information, and ensured that participants would make some errors. On every trial, participants judged which line was longer, as quickly as possible. "Horizontal" or "Vertical" responses were registered by depressing the " $h$ " or "v" keys, respectively, on the keyboard. The inter-trial interval was $500 \mathrm{~ms}$. Participants completed sixteen blocks of 18 trials (288 trials total). In each block, only one individual's face was shown, but its expression changed from trial to trial in quasi-random sequence. Faces were used twice across the entire experiment. At the end of each block, participants were asked, "On how many trials do you think that you responded correctly, out of 18 trials?" This provided a rough estimate of how aware participants were of their accuracy, to verify that the task engaged error-monitoring processes. Within each block, each face expression, linelength pair, and correct answer $(\mathrm{H}$ or $\mathrm{V})$ was equally probable. All of these factors were combined in randomized order. A practice block of 36 trials, with visual feedback on each trial, preceded the experimental task. Only correct trials were used for analysis-that is, PES was not assessed when an error was followed by another error. For each emotion, we calculated the PES by subtracting the mean reaction time of correct trials preceded by correct trials (PCC-RT) from the mean reaction time of correct trials preceded by incorrect trials (PEC-RT). This method 
has been used in previous studies to calculate PES (e.g. Gupta et al. 2009, 2011; Gupta and Kar 2009; Schachar et al. 2004).

\section{Results}

In order to test for specific PES effects, and also for possible speed differences based on current facial expression (e.g. happy-face facilitation), and for possible incongruence effects (i.e. mismatches of prior accuracy and current facial expression valence), we performed a two-way ANOVA on RTs, with expression (happy, sad, neutral) and previous-trial accuracy (post-correct, post-error) as withinsubjects factors. Only correct response trials were included in this analysis. ${ }^{1}$

There was a significant main effect of previous-trial accuracy, $\quad F(1,19)=13.52=\mathrm{MSE}=2570.3, \quad p<.01$, $\eta_{p}^{2}=.416$. Reaction times were longer on correct trials following an error trial (PE-RT, $M=822 \mathrm{~ms}, \mathrm{SD}=100 \mathrm{~ms}$ ) than on correct trials following a correct trail (PC-RT, $M=788 \mathrm{~ms}, \mathrm{SD}=98 \mathrm{~ms}$ ). Thus, critically, the task elicited a PES effect. There was, however, no significant main effect of expression, $F(2,38)=1.74, \mathrm{MSE}=4616.1, p=.188$, $\eta_{p}^{2}=.084$, confirming that the effect was not caused by happy faces (or sad or neutral faces) in general, or by nonneutral expressions more generally.

Importantly, there was a significant interaction of expression and previous-trial accuracy on RT, $F(2,38)=3.18, \operatorname{MSE}=4311.1, p<.05, \eta_{p}^{2}=.144 . \mathrm{A}$ significant difference was found between mean PC-RT and PE-RT on trials with happy expressions, $t(19)=3.28$, $p<.01$. However, the PCC-RT-PEC-RT difference was not significant on trials with sad or neutral expressions ( $p>.23$ for both, see Fig. 2). Thus, participants showed a reliable PES effect only when the post-error distractor had a happy expression. This interaction rules out the possibility that slowing was due to any mismatch between expression and previous-trial accuracy.

Interestingly, PES also varied as a function of emotion expressed in trial $n$ (i.e. the error trial): PES was greater after errors made during an irrelevant happy expression $(M=70 \mathrm{~ms}, \mathrm{SD}=64 \mathrm{~ms})$ than during a sad expression $(M=-16 \mathrm{~ms}, \quad \mathrm{SD}=57 \mathrm{~ms}), \quad t(19)=4.65, \quad p<.001$. There were no other differences. This suggests that concurrent as well as irrelevant happy expressions can modulate PES in a difficult task. Notably, Boksem et al. (2011) did not find this effect, perhaps because their task was much less difficult that the current one.

\footnotetext{
1 There was no significant effect of participant gender on PES $(p=.46)$ nor was there an interaction with facial expression $(p>.08)$. Thus, gender is not considered in any further analyses.
}

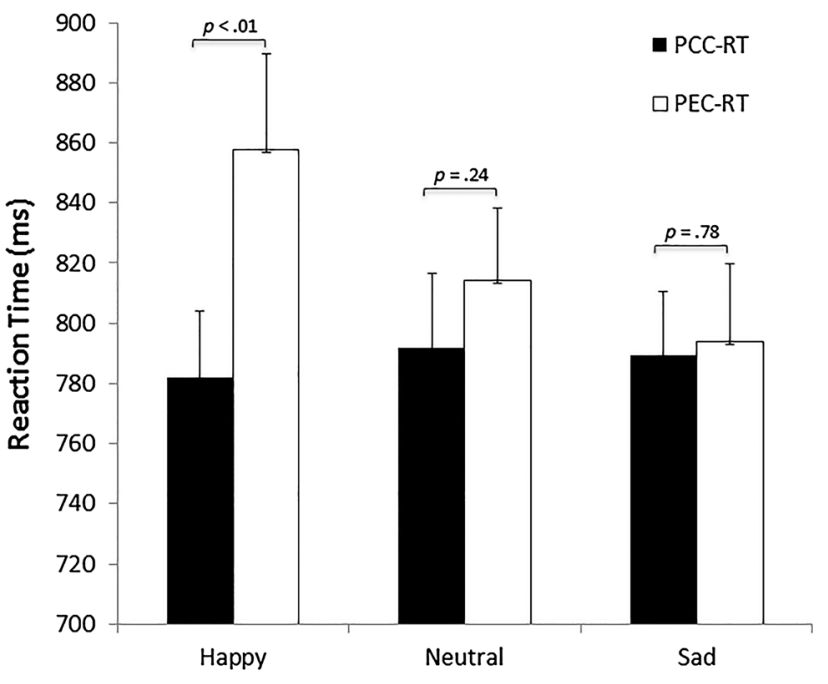

Fig. 2 Mean RT of correct trials following error trials (open bars; PEC-RT) and correct trials (black bars; PCC-RT) for each type of prime. Vertical lines represent \pm 1 SE. Note PCC-RT: correct trials preceded by correct trials; PEC-RT: correct trials preceded by error trials

Evidence reported by Notebaert et al. (2009) suggests that PES might be due to an "oddball effect". If, for example, happy faces rarely followed error trials, the infrequency of that contingency might account for the PES effect. To rule this out, we compared mean percentage of correct responses followed by happy $(M=27 \%$, $\mathrm{SD}=6 \%), \operatorname{sad}(M=26 \%, \mathrm{SD}=7 \%)$, and neutral faces $(M=24 \%, \mathrm{SD}=6 \%)$. A one-way ANOVA with post-error expression (happy, sad, and neutral) as the within-group factor confirmed that these did not differ, $F(2,38)=.925, \mathrm{MSE}=44.4, p=.405, \eta_{p}^{2}=.046$. Thus, PES was not due to an "oddball effect", even though this might sometimes account for PES (or PES-like) effects (Notebaert et al. 2009).

To further show that overall performance was unrelated to facial expression in general, we performed one-way ANOVA on overall RT and on accuracy on correct trials relative to current distractor face expression (happy, sad, or neutral) as a within-group factor. There was no significant main effect of expression on RT, $F(2,38)=.315$, $\mathrm{MSE}=595.5, \quad p=.73, \eta_{p}^{2}=.016, \quad$ or on accuracy, $F(2,38)=1.62, M S E=11.6, p=.21, \eta_{p}^{2}=.079$. Average RTs were very similar across expressions [happy $=804 \mathrm{~ms}, \quad(\mathrm{SD}=99), \quad \mathrm{sad}=798 \quad(\mathrm{SD}=95)$, neutral $=803(\mathrm{SD}=110)]$. Average accuracy was also very similar [happy $=83 \%(\mathrm{SD}=8 \%), \operatorname{sad}=81 \%$ $(\mathrm{SD}=7)$, neutral $=83 \%(\mathrm{SD}=8)]$. This further shows that the dependence of the PES effect on a happy face was not due to a general effect of happy faces on concurrent processing (e.g. a dual-task effect). 
One important question for interpreting the PES effect is whether participants knew (or at least suspected) when they had made errors. Although it is unclear whether PES requires awareness of errors (Navarro-Cebrian et al. 2013), if participants are aware of their accuracy, then errormonitoring and cognitive control processes were engaged while participants performed the task (it does not, however, necessarily indicate that PES entails awareness or metacognitive control processes). To assess this, we considered participants' estimates of their overall accuracy, collected after every block of trials (the task did not allow for obtaining accuracy judgments after every trial). A high positive correlation between participants' actual accuracy $(M=83 \%, \mathrm{SD}=7)$, and their self-estimated accuracy $(M=71 \%, \mathrm{SD}=17)$, would indicate that participants had a robust internal error signal. The correlation was in fact significantly greater than chance, $r=.547, p<.01$. As another, indirect measure of participant error-processing, we also compared the mean percentage of errors immediately following error trials to the mean percentage of correct trials immediately following the error trials. Participants made significantly more correct responses $(M=14 \%, \mathrm{SD}=4 \%)$ following error trials than errors $(M=4 \%, \quad \mathrm{SD}=3 \%)$ following the error trials, $t(19)=18.34, p<.001$. To further confirm, we also compared the mean percentage of errors immediately following error trials to the mean percentage of error trials immediately following the correct trials. Participants made significantly less error trials following error trials $(M=4 \%, \mathrm{SD}=3 \%)$ compared to errors following correct trials $(M=13 \%, \quad \mathrm{SD}=4 \%), \quad t(19)=16.81$, $p<.001$. In other words, PES might be related to more cautious or thorough task-related processing after making an error. This further suggests that participants were monitoring and adaptively responding their own errors.

Also, to ensure that the task did not become so easy with practice that PES was a weak dependent measure, we examined accuracy in the last two blocks of trials. Accuracy averaged $86 \%(\mathrm{SD}=9 \%)$, indicating that practice did not cause ceiling effects. Thus, the entire task-elicited errors and subjects were still making errors even at the end of the task.

\section{Discussion}

The results suggest that in some contexts, a perceived smiling face does not facilitate task performance. If the positive valence of the cue is inconsistent with participants' self-evaluation of their prior response accuracy, the expression distracts from concurrent task processing. The results replicate previous findings that people sometimes slow their responses after making an error. However, the results show that this slowing can be modulated by social information-specifically, a response-incongruent but taskirrelevant emotional cue.

The results are consistent with the error-monitoring model proposed by Gehring et al. (1993), which predicts that processing time in post-error trials is affected by the degree of conflict during the error, such that greater conflict results in more PES. Gehring et al. (1993) suggested that the mechanism underlying this effect is a feedback loop involving conflict detection and processing, and response control mechanisms. This loop temporarily reduces response priming during periods of high conflict, resulting in slower responses. In contrast, the loop primes responses during periods of low conflict, resulting in faster responses without accuracy costs (see Botvinick et al. 2001). Errors are therefore associated with a high degree of cognitive conflict. In this task, the conflict was apparently prolonged when a happy face appeared just after an error, though the face was clearly independent of task performance, and therefore not explicitly perceived as feedback. It is possible that the incongruency of the valence of the facial expression increased the conflict signal. According to Gehring et al.'s (1993) theory, the error signal is proportional to the degree of conflict, and the magnitude of PES should co-vary with the amount of conflict (see Botvinick et al. 2001, for a computational simulation of this effect). However, incongruency due to presentation of a sad face following a correct response did not cause slowing. Thus, incongruency per se does not necessarily generate a conflict signal adequate to slow responding. One reason is that without a clear internal error signal, the mere perception of an incidental negative expression might not generate a conflict signal large enough to engage the feedback loop. This makes sense because it is inevitable that we sometimes witness others' negative emotional expressions, even when we are not making errors, or being evaluated, or even interacting with the person making the expression. Even when we witness negative expressions that are related to our performance, they should not always generate an errorcorrection signal: for example, when playing a competitive game, our successes might elicit negative emotional expressions from our competitor. Such responses are not unexpected or incongruent in light of the social-motivational context, and should not elicit PES. That is, successful moves in a competitive game are not errors and should not generate self-correction processes, even though they might elicit negative reactions from other players. These considerations might explain why there was no slowing when a sad face followed correct responses.

However, in other situations, incongruent internal signals and social cues might generate additional processing: specifically, in some cases, our errors might elicit positive emotional expressions from others (e.g. when we make a 
mistake during a competition, or an embarrassing gaffe that amuses onlookers). In such cases, our internal negative self-evaluation already primes error-correction control processes, and the discrepant emotional cues from others (e.g. laughter) might enhance or strengthen the error signal, or even provide a punishment signal that facilitates further behavioural adjustment, including PES.

This explanation could be challenged by an alternative hypothesis that PES is an enhanced orienting response to rare or discrepant events (Notebaert et al. 2009). The current results are not, however, consistent with that model: happy faces were no less common after errors than sad or neutral faces. Moreover, error-processing accounts of PES such as Botvinick et al (2001) predict post-error improvement of accuracy (PIA) due to adaptive cognitive control, whereas oddball-orienting does not predict PIA. Notably, PIA, was observed in the present study, further suggesting that oddball-orienting cannot explain the current effects. However, a more specific version of the oddball-orienting hypothesis is possible: perhaps the happy faces had some additional feature that influenced post-error RTs. For example, in the present stimuli, only the happy faces displayed teeth. An orienting account of PES might suggest that the infrequent conjunction of a prior error, and this expression-specific feature jointly generated an orienting response that elicited more cautious behaviour. However, this account is not supported by the lack of evidence that happy faces were any more salient than other expressions: there were no expression-based differences in overall RT, in PC-RT, or in concurrent accuracy. Also, we know of no prior evidence suggesting that in humans, exposed teeth (independent of other features of smiles) are a particularly salient attention-getting cue. Nevertheless, in future studies, it would be ideal to use a wider range of facial expressions with more variable display features (e.g. visibility of teeth), to rule out the possibility that any specific low-level expression-specific feature modulated errorprocessing.

Participants did not show general facilitation of processing due to happy faces. This is interesting because adults sometimes show response facilitation in response to incidental happy facial expressions (Winkielman et al. 1997), and happy faces are detected rapidly even in inattentive conditions (Gupta and Srinivasan 2014; Srivastava and Srinivasan 2010; Srinivasan and Gupta 2010, 2011). The absence of an overall effect of expression confirms that the facial expressions were not explicitly used or perceived as feedback, and that the PES effect was an interaction between error processing and incidental facial expression processing.

In sum, the results of the present study are best understood within the error-monitoring model of Gehring et al (1993). The model stipulates that anterior cingulate cortex
(ACC) has a role in error monitoring. This is supported by the electroencephalograph phenomenon of error-related negativity (ERN). ERN appears selectively on error trials, and typically is strongly related to imputed midline frontal source generators that lie in or near ACC (e.g. Debener et al. 2005; Hoffmann et al. 2013). It has been suggested that ACC output (presumably related to the imputed generators) is a critical component of error-feedback modulation, and therefore of cognitive control (Botvinick et al. 2001). Gehring et al. (1993) showed that the magnitude of ACC activation during errors, and the magnitude of ERN, is positively correlated with the magnitude of slowing on subsequent trials. ERN results also indicate that affective evaluation occurs during error detection (Bush et al. 2000). Interestingly, there is evidence that ERN is responsive to social feedback (Boksem et al. 2010). Thus, error monitoring might be sensitive to facial expression. Although we know of no direct evidence of this, there are several intriguing related results. Liao et al. (2011) found that feedback-related negativity (FRN) is sensitive to both direct and implicit error feedback. Picton et al. (2012) found that in a cooperative task, FRN is elicited not only by a participant's own errors, but also by the partner's errors. Thus, indirect or performance-unrelated social feedback can nevertheless affect error-feedback processing. The current results extend this evidence by showing that a behavioural adaptation to error and/or feedback-PESmight be elicited or strengthened by social cues. In the current paradigm, facial cues did not serve as feedback per se: that is, they were not informative or responsive but random. Relevant to this, de Bruijn et al. (2004) showed enhanced P300 amplitude (associated with unexpected events) in some individuals in response to false feedback. Notably, those individuals also showed behavioural PES. Thus, participants who attributed more meaning to false feedback were more likely to show PES. This supports the hypothesis that task-irrelevant social feedback can have a behavioural effect. However, it also suggests that the effect might be sensitive to individual and contextual differences. For example, the fact that our participants did not receive direct error feedback might explain why PES was only reliable if there was an additional incongruent post-error facial cue. Perhaps in situations that do not elicit strong PES effects, adding an incongruent social cue is sufficient to elicit PES. This interpretation could be tested by measuring ERN magnitude, FRN magnitude, and P300 (to assess incongruency) in response to errors, to social feedback, and to both.

These results raise intriguing questions about the effects of social information processing on error processing, feedback processing, and adaptive cognitive control. It would be ideal in future studies to measure face-processing components (see Luo et al. 2010) as well as the 
aforementioned ERP phenomena and behavioural indices such as PES. This is because facial expression processing seems to involve some different cortical networks than those involved in error processing, and in fact negative and positive facial expressions are processed in somewhat different cortical and subcortical pathways (e.g. Luo et al. 2010; Pessoa et al. 2002; Pessoa 2009). By simultaneously measuring both facial-processing and cognitive control EEG dynamics, we might gain insight at to why, for example, PES was facial expression-specific. Regardless of this, if our interpretation is correct, perhaps social cues other than facial expression (e.g. prosodic cues in vocal feedback; surprised expressions) can enhance PER or other error-processing effects.

Another question concerns the strength of the internal error signal, and the importance of this variable in PES. In the present study, we did not give explicit feedback on error trials because the PES effect is brief, and explicit post-response feedback would likely have masked the effect. Thus, we do not know whether participants' internal error signals were accurate on a trial-by-trial basis. However, participants were fairly accurate in estimating their overall accuracy, so it is likely that errors usually, if nor always, generated error signals. Nonetheless, it would be interesting in future studies to compare explicit and implicit error feedback, or vary the difficulty of the task or the frequency of errors (see Liao et al. 2011; Notebaert et al. 2009).

A final question is why, given that there was contextually specific automatic processing of happy faces, was there no evidence of an effect of sad faces? That is, there was significant PES only in the context of happy post-responses face, not in the context of sad or neutral faces. A plausible explanation is that our subjects did not receive feedback on every trial, and the task was difficult enough that subject might not have been certain of their accuracy on every trial (even though they were above chance in judging their overall accuracy). This might have weakened the overall PES effect and made it sensitive to other feedback elements, such as an incongruent positive expression. Although one might expect sad faces to also support PES, it has been argued that among negative expressions, social evaluation is less associated with sadness than with anger, contempt, or disgust (e.g. Baumeister et al. 2007). This argument is supported by evidence that ERN (error-related negativity) amplitudes was larger when the stimuli were incidental disgusted faces than when they were sad (or happy) faces (Boksem et al. 2011). Thus, it seems that disgust faces are stronger error-correction signals. Followup studies could address the degree to which a variety of negative, positive, and ambiguous facial expressions can serve as social cues that trigger feedback-related errorprocessing effects.
In summary, this study provides first evidence that PES can occur if an incongruent but task-irrelevant social cue, a happy facial expression, coincides with an internal error signal. The finding adds to our understanding of the functions of implicit, automatic processing of facial affect. In addition, the results are among the first to show how social affect cues can influence cognitive control (see also e.g. Park and Kitayama 2014; Schwarz et al. 2013). The findings have broader implications for our understanding of interactions between error-processing mechanisms and social affect processing. For example, they suggest that positive performance feedback (smiles, praise) requires additional processing when the feedback occurs in difficult, error-prone tasks. This suggestion, if valid, might have implications for the practical use of positive social feedback in challenging vocational or educational settings. This is a complex but farreaching question for future exploration.

Acknowledgments This research was supported by a Grant from the National Science Foundation (SBE-0542013) to the Temporal Dynamics of Learning Center. The authors thank Piotr Winkielman and Shirlene Wade for helpful feedback.

\section{References}

Batty M, Taylor MJ (2003) Early processing of the six basic facial emotional expressions. Cogn Brain Res 17:613-620

Baumeister RF, Vohs KD, DeWall CN, Zhang LQ (2007) How emotion shapes behavior: feedback, anticipation, and reflection, rather than direct causation. Personal Soc Psychol Rev 11:167-203

Boksem MA, Kostermans E, De Cremer D (2010) Failing where others have succeeded: medial frontal negativity tracks failure in a social context. Psychophysiology 48:973-979

Boksem MA, Ruys KI, Aarts H (2011) Facing disapproval: performance monitoring in a social context. Soc Neurosci 6:360-368

Botvinick MM, Braver TS, Barch DM, Carter CS, Cohen JD (2001) Conflict monitoring and cognitive control. Psychol Rev 108:624-652

Bush G, Luu P, Posner MI (2000) Cognitive and emotional influences in anterior cingulate cortex. Trends Cogn Sci 4:215-222

Danielmeie C, Ullsperger M (2011) Post-error adjustments. Front Psychol 2:233

de Bruijn ERA, Mars RB, Hulstijn W (2004) It was not me ... or was it? How false feedback affects performance. In: Ullsperger M, Falkenstein M (eds) Errors, conflicts, and the brain. Current opinions on performance monitoring. MPI of Cognitive Neuroscience, Leipzig, pp 118-124

Debener S, Ullsperger M, Siegel M, Fiehler K, von Cramon DY, Engel AK (2005) Trial-by-trial coupling of concurrent electroencephalogram and functional magnetic resonance imaging identifies the dynamics of performance monitoring. J Neurosci 25:11730-11737

Farroni T, Menon E, Rigato S, Johnson MH (2007) The perception of facial expressions in newborns. Eur J Exp Psychol 4:2-13

Funder DC (1987) Errors and mistakes: evaluating the accuracy of social judgment. Psychol Bull 101:75-90

Gao X, Maurer D (2009) Influence of intensity on children's sensitivity to happy, sad, and fearful facial expressions. J Exp Child Psychol 102:503-521 
Gehring WJ, Goss B, Coles MGH, Meyer DE, Donchin E (1993) A neural system for error detection and compensation. Psychol Science 4:385-390

Gupta R, Kar BR (2009) Development of attentional processes in normal and ADHD children. Prog Brain Res 176:259-276

Gupta R, Srinivasan N (2014) Only irrelevant sad but not happy faces are inhibited under high perceptual load. Cogn Emot 7:1-8

Gupta R, Kar BR, Srinivasan N (2009) Development of task switching and post-error slowing in children. Behav Brain Funct 5:38

Gupta R, Kar BR, Srinivasan N (2011) Cognitive-motivational deficits in ADHD: development of a classification system. Child Neuropsychol 17:67-81

Herba CM, Phillips M (2004) Annotation: development of facial expression recognition from childhood to adolescence: behavioural and neurological perspectives. J Child Psychol Psychiatry 45:1185-1198

Hoffmann S, Labrenz F, Themann M, Wascher E, Beste C (2013) Crosslinking EEG time-frequency decomposition and fMRI in error monitoring. Brain Struct Funct 1:11

Jiang Y, He S (2006) Cortical responses to invisible faces: dissociating subsystems for facial-information processing. Curr Biol 16:2023-2029

Jones LB, Rothbart MK, Posner MI (2003) Development of executive attention in preschool children. Dev Sci 6:498-504

Korban L, Pourtois G (2014) Brain systems underlying the affective and social monitoring of actions: an integrative review. Neurosci Biobehav Rev. doi:10.1016/j.neubiorev.2014.02.014

Liao Y, Gramman K, Feng W, Deák GO, Li H (2011) This ought to be good: brain activity accompanying positive and negative expectations and outcomes. Psychophysiology 48:1412-1419

Lundqvist D, Flykt A, Öhman A (1998) The Karolinska Directed Emotional Faces-KDEF, CD ROM from Department of Clinical Neuroscience, Psychology section, Karolinska Institutet, ISBN 91-630-7164-9

Luo W, Feng W, He W, Wang NY, Luo YJ (2010) Three stages of facial expression processing: ERP study with rapid serial visual presentation. Neuroimage 49:1857-1867

Marco-Pallares J, Camara E, Munte TF, Rodriguez-Fornells A (2008) Neural mechanisms underlying adaptive actions after slips. J Cogn Neurosci 20:1595-1610

Navarro-Cebrian A, Knight RT, Kayser AS (2013) Error-monitoring and post-error compensations: dissociation between perceptual failures and motor errors with and without awareness. J Neurosci 33:12375-12383

Norman D, Shallice T (1986) Attention to action: willed and automatic control of behavior. In: Davidson RJ, Schwartz GE, Shapiro D (eds) Consciousness and self-regulation. Plenum Press, New York

Notebaert W, Houtman F, Opstal FV, Gevers W, Fias W, Verguts T (2009) Post-error slowing: an orienting account. Cognition 111:275-279

Park J, Kitayama S (2014) Interdependent selves show face-induced facilitation of error processing: cultural neuroscience of selfthreat. Soc Cogn Affect Neurosci 9:201-208
Pessoa L (2009) How do emotion and motivation direct executive control? Trends Cogn Sci 13:160-166

Pessoa L, McKenna M, Gutierrez E, Ungerleider G (2002) Neural processing of emotional faces requires attention. PNAS 99:11458-11463

Picton L, Saunders B, Jentzsch I (2012) "I will fix only my own mistakes": an ERP study investigating error processing in a joint choice-RT task. Neuropsychologia 50:777-785

Pizzagalli DA, Lehmann D, Hendrick AM, Regard M, PascualMarqui RD, Davidson RJ (2002) Affective judgments of faces modulate early activity (approximately $160 \mathrm{~ms}$ ) within the fusiform gyri. Neuroimage 16:663-677

Ridderinkhof KR (2002) Micro- and macro-adjustments of task set: activation and suppression in conflict tasks. Psychol Res 66:312-323

Schachar R, Chen S, Logan GD, Ornstein TJ, Crosbie J, Ickowicz A, Pakulak A (2004) Evidence for an error monitoring deficit in attention deficit hyperactivity disorder. J Abnorm Child Psychol 32:285-293

Schacht A, Dimigen O, Sommer W (2010) Emotions in cognitive conflicts are not aversive but are task specific. Cogn Affect Behav Neurosci 10:349-356

Scherer KR (2001) Appraisal considers as a process of multilevel sequential checking. In: Scherer KR, Schorr A, Johnstone T (eds) Appraisal processes in emotion: theory, methods, research. Oxford University Press, Oxford, pp 92-120

Schlenker BR, Leary MR (1982) Social anxiety and self-presentation: a conceptualization model. Psychol Bull 92:641-669

Schneider W, Eschman A, Zuccolotto A (2002) E-Prime user's guide. Psychological Software Tools, Pittsburgh

Schwarz KA, Wieser MJ, Gerdes AB, Mühlberger A, Pauli P (2013) Why are you looking like that? How the context influences evaluation and processing of human faces. Soc Cogn Affect Neurosci 8:438-445

Shackman AJ, Salomons TV, Slagter HA, Fox AS, Winter JJ, Davidson RJ (2011) The integration of negative affect, pain and cognitive control in the cingulate cortex. Nat Rev Neurosci 12:154-167

Srinivasan N, Gupta R (2010) Emotion-attention interactions in recognition memory for distractor faces. Emotion 10:207-215

Srinivasan N, Gupta R (2011) Global-happy and local-sad: perceptual processing affects recognition of distractor emotional faces. Q J Exp Psychol 64:425-433

Srivastava P, Srinivasan N (2010) Time course of visual attention with emotional faces. Atten Percept Psychophys 72:369-377

Vuilleumier P, Armony JL, Driver J, Dolan RJ (2001) Effects of attention and emotion on face processing in the human brain: an event-related fMRI study. Neuron 30:829-841

Walden T, Ogan T (1988) The development of social referencing. Child Dev 59:1230-1240

Winkielman P, Zajonc RB, Schwarz N (1997) Subliminal affective priming resists attributional interventions. Cogn Emot 11:433-465 